\title{
La investigación psicológica de la inteligencia humana: balance del siglo en la transición del milenio*
}

\author{
Maria José Miranda \\ Universidad de Lisboa \\ Lisboa, Portugal
}

La problemática de la inteligencia humana cruza la historia del pensamiento occidental y, por supuesto, toda la historia de la psicología. Las cuestiones conceptuales son analizadas a lo largo del siglo XX, y las grandes teorías presentadas bajo cuatro paradigmas: el neurobiológico, el diferencial, el psicogenético y el informacional. La investigación del siglo XXI recuperará con seguridad caminos abiertos en los años noventa, sobre todo en lo que concierne a la interdisciplinaridad y a los aspectos afectivos y funcionales del comportamiento inteligente. Pero en un nuevo encuadramiento: la globalización, con todos sus retos y riesgos.

historia de la psicología / inteligencia / cognición / evaluación psicológica

\section{Psychological research on human intelligence: 20th century revisited at the dawn of the Third Millennium}

Human intelligence is a recurrent issue along the centuries in western culture. Also, it is a recurrent issue in the history of psychology. Conceptual descriptions along the 20th century are analysed, and main paradigms (biological, differential, genetic, computational) examined. Research on human intelligence in the third millennium will focus upon paths definitely open in the 90s, such as an interdisciplinary approach and emotional and functional aspects of intelligent behavior. The general framework will be, however, new: the globalization, its challenges and risks.

history of psychology / intelligence / cognition / psychological assessment \& evaluation

* Conferencia presentada en la Universidad de Lima, el 6 de octubre de 1999.

Traducción del portugués por Ramón León.

Dirección de la autora: mjmiranda@reitoria.ul.pt 
La inteligencia humana ocupa un lugar relevante en la historia del pensamiento occidental. El estudio de la inteligencia humana se halla íntimamente vinculado con el desarrollo de la psicología.

Ninguna de las dos afirmaciones es pacífica. Y más aún, hablar de inteligencia "humana" presupone que el atributo no es exclusivo de la especie. Sin entrar en los aspectos polémicos, una clarificación todavía se impone.

Primero, el lugar de relieve en la historia del pensamiento occidental. Fueron los griegos, Aristóteles (384-322 a.C.) incluso, quienes distinguieron entre orexis (deseo) y dianoia (intelecto). Cicerón (106-43 a.C.), por su parte, tradujo los términos griegos que designaban la facultad humana de pensar por intelligentia, palabra compuesta por inter (entre) y legere (relacionar, establecer relaciones, escoger).

En el lenguaje común, como en la experiencia colectiva, se considera un caballo como más inteligente que una gallina o un cocodrilo. La fisiología nos ha enseñado la importancia de la relación entre el volumen/peso del cerebro (y del área cortical) y el volumen/peso del cuerpo en las diferentes especies: baja en el elefante, por ejemplo; pero muy alta en el ser humano. Pero el sentido común -que también es importante en la ciencia- recorre en este caso otra vía, pues destaca (tanto en el ser humano como en otros animales) la capacidad adaptativa, la capacidad de aprender, e incluso la capacidad de expresar afectos.

Pásese ahora al estudio de la inteli- gencia en su relación con el desarrollo de la psicología. Si a lo largo de siglos, la filosofía estuvo ligada con la teología (philosophia ancilla theologiae), una circunstancia semejante ocurrió con la psicología: su ligazón a la filosofía, aunque no tanto en términos de ancilla (esclava). La separación se dio con el advenimiento de la ciencia experimental, de laboratorio. Por supuesto que no es de extrañar que la historia de la psicología enfatice la investigación psicofísica en el siglo XIX: de Weber (1795-1878) y Fechner (18011887) a Wilhelm Wundt (1832-1920), con la publicación Elementos de la psicología fisiológica (1874) y la fundación del Laboratorio de Psicología Experimental en la Universidad de Leipzig (en 1879, cuatro años después de ocupar la cátedra de Filosofía). Wundt fue el primero en ser considerado "psicólogo" por sus iguales, y por su laboratorio pasaron muchos científicos ilustres de la época (Donders, Cattell, Baldwin, Stanley Hall, etc.).

Ni Wundt ni los psicofísicos se interesaron nunca por la inteligencia. Por el contrario, su investigación incidía sobre procesos simples (sensoriales y motores, procesos moleculares), sobre la búsqueda de consistencias, de leyes ${ }^{1}$. Habrá que esperar el trabajo de discípulos de Wundt, como James McKeen Cattell (1860-1944), fundador de The

1 Ya para Aristóteles, el padre del pensamiento occidental, sólo hay ciencia de lo universal. La ley es por definición un universal. 
Psychological Corporation y primer editor de revistas de gran prestigio como Psychological Review y Science, para quienes procesos simples, estudiados en el laboratorio, se vuelven indicadores de procesos complejos (molares), la inteligencia. En la ciencia, y lógicamente también en la ciencia psicológica, la medición es condicionada por la tecnología disponible.

En 1859 Charles Darwin (1809-1882) publica su Origen de las especies, que revoluciona la intelligentsia de su época. Así, el antropocentrismo de la cultura occidental sufre uno de sus tres clásicos golpes: los otros dos provendrían de Copérnico y de Freud. Personalmente, añado un cuarto golpe: el de Skinner y el concepto de aprendizaje como modelación recíproca individuomedio. La llamada revolución copernicana desalojó al hombre del centro del universo; la teoría de la evolución lo reubicó en el mundo de la naturaleza; el inconsciente lo reveló en toda su plenitud; el concepto skinneriano introdujo las consecuencias del comportamiento en el desarrollo individual. Pero, lo que nos interesa aquí es Darwin y la importancia en la teoría de la evolución de la transmisión genética.

Su primo, sir Francis Galton (18221911), quien se interesó por la herencia $^{2}$ de la inteligencia. En 1869 publicó Genio hereditario, obra en la cual de-

2 Aunque Galton desconociera las leyes de la genética que, descubiertas por el solitario monje agus- fine la genialidad en términos estadísticos: la genialidad significa sobrepasar en un variado número de mediciones (físicas, sensoriales, motoras y otras) la frontera que separa una pequeña proporción de la mayoría de la población. Una definición estadística (frecuencia de la ocurrencia en la población general) implica medir diferencias individuales y resumir las observaciones: medida, variabilidad interindividual y métodos estadístico-matemáticos de análisis de datos. En otras palabras, supone instrumentos de medición, un gran número de observaciones y análisis exploratorios y confirmatorios. La idiosincrasia es comprendida por la comparación con la población a la cual el individuo pertenece. ¿Qué mide Galton? Los procesos simples (sensoriales, perceptivos, motores), para los que disponía de instrumentos, pero también la imaginación (vía la asociación de palabras) y la memoria (vía la flexibilidad y la precisión de las imágenes).

En 1896 Binet (1857-1911) publica, con Henri, el artículo "La psychologie individuelle": las diferencias individuales son más estables y consistentes en los procesos superiores -vinculados con la adaptación a lo cotidiano- que en los procesos simples, y aquéllos tienen que ser evaluados por tareas relativamente complejas. Es también Binet el autor del primer instrumento psico-

tino Mendel en 1866, sólo fueran conocidas en 1900. 
métrico: la Escala Métrica para la Inteligencia de los Niños, en 1905. El contexto social es la explosión que ocurre como consecuencia de la escolaridad obligatoria, lo que lleva a la constitución de una comisión, presidida por el propio Binet, encargada de distinguir a los niños con dificultades escolares, para apoyo suplementario favorecedor del éxito ${ }^{3}$.

El funcionamiento cognitivo global (la inteligencia o aptitud general) es por naturaleza un indicador muy general del comportamiento (desempeño). Demasiado general, tanto para el pronóstico (escolar, profesional, salud mental) como para la comprensión del individuo y la relación de ayuda que caracteriza la intervención del psicólogo. De ahí la necesidad de dilucidar "segmentos" cognitivos de la inteligencia (genéricamente designados como aptitudes), de "segmentos" no cognitivos (desde las relaciones interpersonales a otros rasgos de personalidad) y del descubrimiento de otras dimensiones del comportamiento.

La idiosincrasia no se compadece con las limitaciones metodológicas y tecnológicas: el funcionamiento cognitivo es un aspecto importante de la singularidad -de la personalidad-, que funciona en conjunción con una multiplicidad de otros aspectos (dimensiones de la

3 El propósito era, así, la promoción de la igualdad de oportunidades, hecho frecuentemente escamoteado en la crítica a los tests psicológicos. personalidad) y con los retos del medio. El concepto de interacción es nuclear en los más variados niveles.

Lo que la investigación en psicología de la inteligencia humana habrá perdido (en mi opinión ha ganado valor acrecentado) en protagonismo, significa el mismo avance de la ciencia psicológica.

Estos hombres (y también nosotros), ¿sabían lo que es la inteligencia? Preguntado un día, Binet dijo que la inteligencia era lo que medía su escala. Esta es la primera definición operacional de la inteligencia. Pero Binet (1910) escribiría más tarde: "dirección, comprensión, invención, crítica", l'intelligence tient dans ces quatre mots.

¿Y nosotros? Hay un acuerdo tácito: la inteligencia es el atributo responsable de las diferencias individuales de competencia de los niños y de los adultos en los dominios del aprendizaje, de los conocimientos, de la eficacia del comportamiento en situaciones nuevas o problemáticas (Miranda, 1987). Se vincula con la adaptación en sentido amplio. Y la cualidad de la adaptación es crucial en la vida de los individuos y de las sociedades.

Pero si no sabemos totalmente lo que es la inteligencia, sí reconocemos con facilidad cuándo es que ella falta. Esto tampoco es nuevo: la psicopatología francesa del siglo XIX (Esquirol, 17721840; Séguin, 1812-1880) se dedicó al estudio de la deficiencia mental y de sus correlatos, y utilizó criterios psico- 
lógicos de diagnóstico y de tratamiento (entrenamiento del lenguaje, del autocontrol, de las capacidades sensoriales y motoras).

\section{EL CONCEPTO}

En 1921, los editores del Journal of Educational Psychology organizaron un simposio sobre la inteligencia y su medición. Las preguntas planteadas a los especialistas fueron las siguientes: a) ¿qué es la inteligencia y cómo puede ser medida?; y, b) ¿cuáles son los próximos pasos en la investigación? (Thorndike et al., 1921). Catorce especialistas presentaron definiciones de la inteligencia humana (otros se centraron, sin embargo, en la segunda pregunta), entre ellas: aprender y adaptarse al medio (S. S. Colvin); la capacidad de aprender y de aprender con la experiencia (W.F. Dearborn); el mecanismo biológico responsable de la integración de los estímulos y de su efecto unificado en el comportamiento (J. Peterson); la capacidad de adaptación a situaciones nuevas (R. Pintner); la aptitud para pensar abstractamente (L. M. Terman); la capacidad para adquirir capacidad (H. Woodrow).

Del análisis de las respuestas (Sternberg \& Detterman, 1986) emergen grandes líneas comunes, cuyos porcentajes se presentan seguidamente: raciocinio abstracto (57\%), capacidad de aprender $(29 \%)$, adaptación al medio (29\%), mecanismos fisiológicos (29\%), procesamiento de información $(21 \%)$, comportamiento eficaz (21\%), emociones/motivación (7\%); $\mathrm{y}$, conocimientos, (7\%).

En 1986 Robert Sternberg \& Douglas Detterman replicaron el simposio de 1921, con 24 especialistas (Sternberg \& Detterman, 1986). A continuación algunos ejemplos de las definiciones: la cualidad adaptativa del comportamiento; en la especie humana la combinación de aptitudes y conocimientos exigidos, promovidos y recompensados en la cultura a la cual se pertenece (A. Anastasi); el conjunto de aptitudes por las cuales los individuos alcanzan los objetivos racionalmente escogidos en el medio en el que viven (J. Baron); la competencia generalizada en los dominios académico, práctico y social (J. Carroll); la propiedad del sistema nervioso central responsable de las diferencias individuales (H.J. Eysenck); la competencia de cognición intelectual (distinta de la cognición emocional) (R. Glaser); el autogobierno mental (R. Sternberg).

Usando la misma taxonomía del análisis de frecuencias (Sternberg \& Detterman, 1986), los resultados son los siguientes: raciocinio abstracto $(50 \%)$, capacidad de aprender (17\%), adaptación al medio (13\%), mecanismos fisiológicos $(8 \%)$, procesamiento de información (21\%), comportamiento eficaz (21\%), emociones/motivación (4\%); y, conocimientos $(21 \%)$.

El pensamiento (raciocinio abstracto) destaca como la referencia mediana de los especialistas en psicología, intere- 
sados en la inteligencia humana distanciados a lo largo de tres generaciones. Dos notas: la referencia de la eminente psicóloga norteamericana Anne Anastasi a la especie humana (lo que naturalmente supone la presencia de inteligencia en otras especies); los indicadores adaptación, eficacia (del comportamiento), mecanismos fisiológicos, $\mathrm{y}$ emociones/motivación. La primera fue ya mencionada; la segunda será retomada más adelante.

Una taxonomía común a las definiciones/descripciones de la inteligencia (y que se remonta a Vernon, 1960; 1979) distingue entre las biológicas y las psicológico/operacionales. Es por demás conocida la formulación de David Wechsler, "agregado o capacidad global para actuar con finalidad, pensar racionalmente y proceder con eficiencia en relación con el medio" (Wechsler, 1958; Matarazzo \& Wechsler, 1972). Mucho menos conocida es, sin embargo, otra formulación suya: "capacidad del individuo para comprender el mundo y los recursos de que dispone para enfrentar a sus desafíos" (Wechsler, 1975).

Tradicionalmente, en una perspectiva amplia, las descripciones/definiciones biológicas de una manera o de otra acentuarían el potencial genético, centrándose en el sistema nervioso central y particularmente en el neocortex (y, en tiempos más recientes, también en el cortex límbico). El acento de las descripciones/definiciones psicológico/ operacionales será colocado también de una manera u otra en la evaluación. Un terreno común, sin embargo: la interacción con el medio. En síntesis, se trataría de acentuación, todavía y siempre en la óptica interaccionista, de genotipo $v s$. fenotipo, o en la formulación que prefiero, natura $v s$. nurtura.

Aquí se imponen tres consideraciones. Pero, antes, la demarcación de la perspectiva tradicional referida. La psicología no tendrá (¿aún?) desafortunadamente una concepción de la persona, pero, definitivamente, la caracteriza en cuanto ciencia del comportamiento una concepción holística de la personalidad, un todo, una totalidad (Reuchlin, 1995) bio-psico-social. Biológico y psicológico significan distinciones metodológicas, convenientes para la démarche científica. Nada más. Pero en el tema que nos ocupa no hay definición/descripción psicológica que no contenga, aunque no haga una referencia explícita, lo biológico, así como no hay definición/descripción biológica que no reporte lo psicológico. ¿Por qué no hay? Porque no podría haberlas.

Volviendo a las consideraciones antes aludidas. La primera es la referida al genotipo y a la formulación lapidaria de Konrad Lorenz: el genoma es un conjunto de informaciones codificadas (Lorenz, 1973). ¿Cuál es el agente de decodificación? El medio. El genoma prefigura los límites del desarrollo; el medio proporciona $-\mathrm{o}$ impide- dicho desarrollo.

El desciframiento del genoma huma- 
no, ¿será una segunda revolución copernicana? Sí, pero con una diferencia esencial con respecto a la primera, por los riesgos incalculables para el más rico patrimonio de la racionalidad y de la persona: la libertad y la dignidad humanas $^{4}$.

La segunda consideración, la distinción entre rasgos no-cognitivos y cognitivos de la personalidad. Los primeros son de naturaleza bipolar (introversión-extraversión y un continuum en el cual la evaluación sitúa al individuo); los segundos, entre los que se incluye la inteligencia, son de naturaleza unidireccional (y la evaluación traduce una posición relativa).

La tercera se refiere a Wechsler, una de las mayores autoridades en la psicología de la inteligencia humana: en su ya citado artículo de 1975, el autor se refiere a los aspectos conativos y afectivos de la inteligencia humana, en los que se encuentran aprender y comprender los valores (sociales, éticos, estéticos). Y va más allá: el eminente autor de los tests de inteligencia, desde la primera infancia hasta la tercera edad, afirma que "aquello que es medido por los tests (aptitudes) no se identifica con [no agota] aquello que los tests miden (la inteligencia)".

4 Esta afirmación anticipó en varios meses, y en el mismo sentido, los grandes debates corrientes sobre la manipulación genética a nivel humano.

\section{LOS PARADIGMAS}

En otro estudio (Miranda, 1987) se trataron tres grandes paradigmas de la evaluación de la inteligencia humana: el diferencial, el constructivista y el informacional (o cognitivista). Serán aquí tratados desde otra óptica, y naturalmente también el paradigma neurobiológico.

Una precaución inicial: los paradigmas son por naturaleza metáforas, por la riqueza de la problematización que contienen y para la cual la ciencia procura respuestas. Robert Sternberg enumera una variedad de metáforas en la psicología de la inteligencia humana: la geográfica (diferencias inter e intraindividuales), la computacional (procesamiento de información), la biológica (anatomía, fisiología del cerebro), la epistemológica (la lógica formal y la filosofía del conocimiento), la antropológica (la diversidad cultural), la sociológica (el proceso de socialización) y la sistémica (metateorías, generalmente plurimetafóricas).

El paradigma neurobiológico cae en la categoría biológica; el diferencial en la geográfica, el constructivista en las categorías biológica y epistemológica, y ambos también en la antropológica; el informacional en la computacional y en la sistémica. La metáfora sociológica encuadra fundamentalmente los trabajos de Vygotsky (Vygotsky, 1978) y tiene a su exponente en Feuerstnein 1979) (Sternberg, 1990). 


\section{El paradigma neurobiológico}

El paradigma neurofisiológico es, con certeza, el más antiguo: Hipócrates (460-377 a.C.) se refirió a la cabeza, al cerebro, como sede del pensamiento. La teoría frenológica de Franz Joseph Gall (1758-1828) tuvo más impacto en la creencia popular que lo que tuvo en la ciencia. Según ella, la configuración del cráneo reproduciría la estructura del cerebro donde se localizarían las diferentes funciones cognitivas, de modo tal que la palpación de las bolsas craneanas ofrecería indicaciones sobre la inteligencia. El interés actual de la frenología es sólo histórico. Pero en la creencia popular, al menos en Portugal, la altura de la cabeza es todavía un indicador de nivel de inteligencia ${ }^{5}$.

Una de las más importantes e influyentes teorías biológicas de la inteligencia humana, neuropsicológica en la formulación de su autor, es la de Hebb (1949): de una manera simplificada la inteligencia A significa el potencial innato, esto es, la capacidad del sistema nervioso central que incluye la de su propio desarrollo, que no es observable ni mensurable; la inteligencia $\mathrm{B}$ es el resultado de la interacción de la Inteligencia A con el medio, esto es el fenotipo ${ }^{6}$.

5 A modo de dato histórico, recuérdese que en sus primeros trabajos sobre la inteligencia Binet usó el índice cefálico (perímetro del cráneo) como indicador.

6 Lo que llevaría a Vernon a proponer la inteligencia C, la que miden los tests (Vernon 1961).
En la teoría de Cattell (1971), la inteligencia fluida (gf) es biológicamente organizada, constitucional y significa el funcionamiento intelectual biológicamente determinado (natura). A la inteligencia fluida se contrapone la inteligencia cristalizada (gc), resultado de la aculturación (nurtura) .

Estudios de la actividad cerebral, desde la velocidad de la conducción neuronal a los patrones eléctricos complejos (EEG), y el metabolismo de la glucosa, han abierto vías interesantes (véase la revista Intelligence $\mathrm{y}$, por ejemplo, Barrett \& Eysenck, 1992) para la investigación y para la práctica clínica (Matarazzo, 1992).

En el área de la neurobiología (o de la neuropsicología, como prefiero denominarla), los trabajos del portugués António Damásio y su esposa Hanna (Damásio, 1994; Damásio et al., 1994) y colaboradores, en la Universidad de Iowa, constituyen una referencia ineludible en el fin del milenio. El concepto básico de la obra mundialmente famosa (Damásio, 1994) es relativamente simple, aunque demasiado frecuentemente no entendido ${ }^{7}$ : no hay res cogitans sin res extensa - no hay cogito sin cuerpo- no hay pensamiento sin un sustrato neurobiológico. Pero los auto-

7 En su obra de finales de 1999 Damásio vincula explícitamente y por primera vez el "sentimiento de sí” a la res extensa, al cuerpo (Damásio 1999). La obra es todavía posterior al texto de la conferencia, aquí literalmente reproducido. 
res van mucho más allá: recuperan, a través de la neurociencia, los afectos y las emociones como componentes esenciales de la racionalidad.

\section{El paradigma diferencial}

El paradigma diferencial radica en la evidencia de las diferencias individuales (inter-, intra-, entre grupos). La comprensión de los fenómenos (inteligencia, ansiedad, calidad de vida) pasa por las diferencias y, más, son las diferencias observadas las que legitiman los constructos psicológicos. Una teoría, una ley, pasa por las diferencias; y la idiosincrasia es eso mismo: la diferencia.

El paradigma diferencial es eminentemente evaluativo: la evaluación es el punto de partida (la averiguación de las variabilidades); la evaluación es el punto de llegada (el conocimiento del individuo), y la compartida con el psicólogo, favorecedora del autoconocimiento.

En la psicología de la inteligencia humana los modelos y su operacionalización (las tecnologías de la observación) datan de la transición del siglo. Los dos grandes motores de los desarrollos teóricos (y prácticos) fueron y son la evolución de los métodos de observación y la de los métodos de análisis de datos. De éstos, el análisis factorial ocupa un lugar preeminente en la teoría psicológica (modelos y técnicas).

Los métodos de observación (genéricamente los tests) son la operacionali- zación de un constructo psicológico. La definición conceptual enuncia las dimensiones (inteligencia, memoria, raciocinio verbal/numérico/espacial, discriminación perceptiva, etc.); la definición operacional (la operacionalización, el instrumento) es una muestra de funciones (los ítems del test). La estandarización (reglas de aplicación, puntuación e interpretación) deriva de la observación y análisis de los resultados de la muestra inicial (muestra de estandarización). Una muestra de estandarización es una miniatura de la población; esto es, reproduce la variabilidad de la población en aspectos relevantes para el constructo, de naturaleza biológica (por ejemplo, edad y sexo), social (tales como escolarización o el medio sociocultural) u otra. Los análisis de los resultados incluyen los aspectos metrológicos (distribuciones de medidas, validez, precisión, varianza) y la derivación de las normas (significado, o equivalencia de los resultados brutos). El instrumento validado es usado en la observación de individuos de la misma población: la referencia de la lectura e interpretación del resultado individual, indicador único o múltiple, son las normas (Miranda, 1982, 1983, 1987, 1994, 1996, 1999).

La inmensa variedad de modelos y técnicas (véase Huteau \& Lautrey, 1999; también Anastasi, 1997) deriva fundamentalmente del valor heurístico del propio constructo de inteligencia. ¿Será él socialmente relevante? ¿So- 
cialmente necesario? ¿Y, por lo menos socialmente útil para el individuo (autoconocimiento, capitalización de los puntos fuertes y eventual superación de los puntos débiles) y para la sociedad (el mérito personal es un vector importantísimo de la igualdad de oportunidades)?

¿Y el llamado impacto adverso (desventaja de las minorías -sexuales, étnicas, culturales- tantas veces mayoritarias?

Es un falso problema, un pseudoproblema: primero, porque introduce la desconfianza (sin incluir siquiera el beneficio de la duda) relativa a la competencia (científica, técnica y deontológica) del psicólogo certificado; segundo, porque sólo procedimientos dotados de objetividad son preventivos de injusticias sociales; tercero, por lo que significa de desresponsabilización de prácticas atentatorias de la Declaración Universal de los Derechos Humanos (aprobada en la sesión de Asamblea General de las Naciones Unidas de 1948, y que tiene carácter vinculante para todos los países miembros), y que continúan siendo practicadas, consciente e hipócritamente, en algunos de esos países.

\section{El constructivismo psicogenético}

El paradigma constructivista es indisociable de Jean Piaget (1896-1980), que nos legó una inmensidad de trabajos desarrollados a lo largo de más de medio siglo y de una escuela de expansión mundial. "El zoólogo de forma- ción, epistemólogo por vocación y lógico por método" (Miranda, 1987, p. 36), Piaget situó siempre su investigación en psicología de la inteligencia humana, en el puente virtual entre la biología y la epistemología.

La inteligencia es "el estado de equilibrio al que tienden todas las adaptaciones sucesivas sensorio-motoras y cognoscitivas, bien como los cambios asimiladores y acomodadores entre el organismo y el medio" (Piaget, 1956/ 1947, p. 171), y la investigación psicológica traza "su desarrollo desde las formas elementales a las formas superiores de equilibrio" (pp. 62-63).

Inteligencia es adaptación: en el plano mental se prolonga y concluye el conjunto de procesos adaptativos, cuyo punto de partida son las interacciones entre el organismo y el medio que caracterizan la adaptación biológica. La adaptación es asimilación (el sujeto actúa sobre el medio, en el sentido de aprehensión e incorporación de las instancias del medio) y acomodación (el medio actúa sobre el sujeto, en el sentido de la modificación de las estructuras ya existentes). Asimilación y acomodación son comunes a lo orgánico, a la acción y al pensamiento; invariantes, por tanto, de la vida, y la ley funcional de la inteligencia (Piaget, 1956/1947, 1974).

El desarrollo humano es una sucesión de grandes construcciones (sensoriomotoras, operatorias concretas, operatorias formales). Ya Lavoisier (1743- 
1794) decía que "en la naturaleza nada se pierde ni nada se crea; todo se transforma". En el constructivismo psicogenético el desarrollo es una marcha para el equilibrio, y cada construcción integra y reorganiza, en un plano superior, a las que anteceden. Todo se transforma, por tanto. La invarianza del orden de sucesión y la naturaleza integradora de la marcha hacia el equilibrio permiten la distinción de los períodos de desarrollo de la inteligencia: sensoriomotor, preoperatorio, operatorio concreto, operatorio formal. ¿Y cuáles son los factores (los motores) del desarro1lo? Los clásicos -el patrimonio genético, el medio físico, el medio socialmás la equilibración: el crecimiento, el ejercicio y la experiencia, las interacciones y transmisiones sociales son insuficientes, por sí solos como en conjunto, para explicar la trayectoria dirigida del desarrollo; el mecanismo autorregulador -la equilibración- asegura la convergencia de los efectos de la maduración, de la experiencia física y de la experiencia social (Piaget \& Inhelder, 1970).

La evaluación operacionaliza los conceptos de la teoría genética operatoria, vía las situaciones estandarizadas de observación (llamadas "pruebas operatorias"). Las pruebas piagetianas sirven, también, al entrenamiento cognitivo, en la medida en que constituyen experiencias movilizadoras y amplificadoras de la actividad mental (Miranda, 1987).

\section{El paradigma informacional}

El paradigma informacional se centra en los mecanismos de la cognición; esto es, en el procesamiento de la información (por la persona como por la máquina, la llamada inteligencia artificial). En el estudio de las representaciones y de los procesos entran los correlatos, los componentes y los contenidos cognitivos (Sternberg, 1981; 1990), en los aspectos de consistencia, de variabilidad y de cambio.

En el cuadro del paradigma informacional, los trabajos de R.J. Sternberg son muy destacados. A fines de los años setenta él formula su teoría componencial de la inteligencia humana, que -a mediados de los ochenta- integra, en cuanto subteoría, el modelo triárquico de la inteligencia humana ${ }^{8}$.

La teoría triárquica está constituida por tres subteorías: la componencial, que trata de los mecanismos del comportamiento inteligente (componentes de adquisición, de información y de ejecución de tareas cognitivas, y los metacomponentes, que rigen a las estrategias); la contextual, que trata de la tifipicación, por el medio, del comportamiento inteligente en un determinado contexto cultural (respuestas de adecuación, selección, modelación del medio por parte del individuo); la expe-

8 Ya en los años noventa, Sternberg formula y operacionaliza un nuevo modelo de la inteligencia humana, el modelo del autogobierno mental (Sternberg 1997; Miranda 1994, 1998). 
riencial, que enfoca facetas críticas del comportamiento inteligente como la automatización de mecanismos de procesamiento de información y la adaptación a situaciones nuevas, como resultado de las interacciones (y de su riqueza) individuo-medio (Sternberg, 1985; 1988). La operacionalización del modelo se ha confrontado con dificultades y vicisitudes de variado orden (Sternberg, comunicación personal).

En el paradigma informacional la metodología de evaluación privilegia las tareas de procesamiento de los estímulos en el laboratorio y recurriendo a tecnología altamente sofisticada. Los modelos son frecuentemente o demasiado circunscritos o demasiado generales y no tienen en cuenta la variabilidad, individual como situacional. De ahí el énfasis en los trabajos de Sternberg en la Universidad de Yale, que constituyen, obviamente no la única pero sí una de las más importantes excepciones.

Asimismo, dos anotaciones: el paradigma informacional introduce definitivamente la acentuación de los mecanismos (procesos) básicos de representación/retención/procesamiento de información en la investigación dentro de la psicología de la inteligencia humana (que tradicionalmente privilegia los productos); $\mathrm{y}$, con todas sus ventajas e inconvenientes, integra en la investigación a la inteligencia artificial. Nos interrogamos, con Negroponte, si no estamos a punto de entrar en la era postinformacional, en la era digital. Todos los avances científicos, en todos los tiempos, tienen también su lado sombrío. ¿Será ese el caso? (Negroponte, 1996).

\section{Desafíos de los años noventa}

Los años noventa han sido plenos de desafíos en todos los niveles. En la psicología de la inteligencia humana se presentaron dos grandes retos: el de la operacionalización de la inteligencia emocional, de Goleman; y, el concepto de successful intelligence, de R. Sternberg.

Empecemos por Goleman. A finales de los años veinte, E.L. Thorndike distinguió la inteligencia verbal (la capacidad para tratar con palabras), la inteligencia numérica (la capacidad para tratar con los símbolos numéricos), y la inteligencia social (la capacidad para tratar con las personas) (Thorndike, 1927). Propuesta de modo recurrente bajo diversas conceptualizaciones (genéricamente denominadas aptitudes interpersonales), los estudios de Salovey $\&$ Mayer en los años noventa constituyen un paso importante: la descripción de la inteligencia emocional como un conjunto de autorregulaciones, aprehensión de sí y de los otros, automotivación, dominio de impulsos, control del humor (Salovey \& Mayer, 1990; 1995, citados en Goleman, 1998). El paso gigantesco vino de la neuropsicología de Iowa (A.R. Damásio y colaboradores): el descubrimiento del sustrato 
neurobiológico del procesamiento de las emociones y de la toma de decisiones, en cuanto dimensiones de la racionalidad (Damásio et al., 1994).

Goleman integra y adapta estas formulaciones en su modelo de inteligencia emocional. Él la describe como la conciencia del sí emocional, y la capacidad para enfrentarse a las emociones, a las motivaciones propias, y con las personas (Goleman, 1995), y la operacionaliza en cinco dimensiones: la conciencia de sí, la motivación, el autocontrol, la empatía y las relaciones interpersonales (Goleman, 1995; 1998).

A la neurociencia va a buscar la arquitectura cerebral, la base neuronal: el cerebro primitivo, el cortex límbico (a partir del cual, por obra de la evolución, surgirán las capas superiores, el cortex y el neocortex). De las relaciones entre el "centinela emocional" (la amígdala) y el neocortex emerge la inteligencia emocional.

Así, a la inteligencia racional se contrapone la inteligencia emocional: al cociente intelectual se contrapone el cociente emocional (Goleman, 1995, p. 161).

Pero, ¿qué es una emoción? Etimológicamente significa movimiento, movere. En la terminología de Goleman, es "un sentimiento y un pensamiento que lo acompaña, un estado psicológico y biológico, un conjunto desencadenador de la acción" (ibídem, p. 289). Como por ejemplo, la tristeza, el miedo, la rabia, el placer, la sorpresa, la aversión, la empatía, etc. (ibídem, pp. 289-290).

Para el autor, la educación emocional y la evaluación de la inteligencia emocional constituyen las claves de la psicología aplicada de la inteligencia humana (Goleman, 1998, p. 229) .

La comprensibilidad, la coherencia y la utilidad del concepto - la inteligencia emocional es un concepto familiar, con evidencia anátomo-fisiológica, y es modificable en el sentido de susceptible de educación y entrenamiento- explican el éxito mundial del autor al que no son ajenos, tampoco, el estilo y el lenguaje periodísticos de sus escritos.

Subráyese, con todo, un punto: a Daniel Goleman, ese maestro de la divulgación y de la comunicación, se le debe sin duda la colocación en pie de igualdad de las competencias intrapersonales e interpersonales con las competencias cognitivas y técnicas. El impacto de esa igualdad en la formación a lo largo de la vida es incalculable. Una cosa es cierta, sin embargo: la economía de mercado vive de la competitividad, y la competitividad vive de la iniciativa y creatividad prácticas; la inteligencia emocional es educable y entrenable, y la economía de mercado es la dominante.

Pásese al constructo (un constructo es una construcción, una hipótesis explicativa de lo real; la materia como la energía son constructos) de successful intelligence de Robert Sternberg (Sternberg, 1995; 1996). La traducción 
es difícil: se trata de eficiencia mental en cuanto determinante de éxito individual, de éxito en la vida. Aquí se me ocurre mencionar a san Agustín (354430): la meta del ser humano es la conquista de la felicidad. Se trata de éxito vivido, según los patrones personales coincidentes o no con los estereotipos sociales. Aunque, provisoriamente, traducimos successful intelligence por inteligencia funcional. El individuo funcionalmente inteligente es un emprendedor, en el sentido de adaptación al medio, de modelación y/o selección del medio: a su medida y para alcanzar sus objetivos.

La inteligencia funcional articula tres grandes aspectos de la inteligencia humana (claves en las palabras del profesor de la Universidad de Yale, incluido en la lista de los 100 más importantes científicos estadounidenses): la inteligencia analítica, la inteligencia creativa y la inteligencia práctica (Sternberg, 1996, pp. 47 y 127).

Las claves del éxito son:

- Clave 1. Descubrir buenas soluciones usando la inteligencia analítica (Sternberg, 1996, p. 155).

- Clave 2. Descubrir buenos problemas usando la inteligencia creativa (ibídem, p. 188).

- Clave 3. Implementar las soluciones usando la inteligencia práctica (ibidem, p. 220).

La inteligencia analítica es responsable de la solución de problemas y de la toma de decisiones; la inteligencia crea- tiva "ve" más lejos, genera y ecuaciona ideas nuevas; $y$, la inteligencia práctica las aplica. La inteligencia práctica es "conocimiento tácito" (Sternberg, 1985 , p. 269; 1996, p. 236), el saber cómo relacionarse consigo mismo, el saber cómo relacionarse con los otros, y el saber cómo relacionarse con las tareas (Afonso, 1997, p. 4).

El test triárquico de las aptitudes (Sternberg, 1993) operacionaliza el modelo triárquico de la inteligencia humana (Sternberg, 1985; 1988) funcionalmente (Sternberg, 1996, p. 147; Afonso, 1997, p. 5): las dimensiones componencial, contextual y experiencial -del pensamiento analítico, del pensamiento creativo y del pensamiento prácticoson evaluadas a través de cuestiones simbólicas (palabras, números) y figurativas (diseños), organizadas para edades desde la preescolar hasta la adulta.

La contribución de Sternberg para la moderna psicología de la inteligencia humana es extraordinariamente importante: en poco más de dos décadas de intensa investigación (más de 700 títulos) superó dicotomías y controversias a través de tres modelos integrativos y amplios (en orden cronológico, la teoría triárquica 1985, el autogobierno 1988, y la inteligencia funcional 1996), pero, sobre todo, articulados y de enorme impacto científico y relevancia social. Construyó (los modelos), operacionalizó (los instrumentos de evaluación, el inventario de estilos de pensamiento, el test triárquico de aptitudes), 
y divulgó (una parte sustancial de sus libros más recientes es dirigida también al gran público).

\section{Consideraciones prospectivas}

¿Y el futuro? Acostumbro decir que en el futuro estaremos todos, o casi todos, muertos, para distinguir la futurología, que rechazo, de la anticipación que caracteriza la propia racionalidad.

Dos grandes trayectorias, claramente sin retorno, están trazadas: vivimos en una sociedad de la información y en una metrópoli global. Las tecnologías de la información y de la comunicación (sociedad del conocimiento) y la globalización, en todos los niveles, son las señales distintivas del próximo milenio. La adaptación a ambas es ya hoy una tarea inmensa, en el plano individual como en el colectivo. Pero, como toda adaptación sólo ella es la garante de la sobrevivencia del individuo y de la especie. El siglo XXI impondrá cambios adaptativos cuya previsión es del ámbito de la futurología.

Las tecnologías de la sociedad del conocimiento constituyen instrumentos esenciales de la ciencia y son un puente entre la comunidad científica y la sociedad en general (desde los laboratorios virtuales al reconocimiento público de la ciencia y de la tecnología como factores de promoción del bienestar): la comunicación en segundos en el ámbito planetario; el acceso a la información científica mundial (base de datos, bibliotecas digitales) desde un pe- queño computador casero; la publicación electrónica permanentemente disponible y sin ocupar espacio; la enseñanza-aprendizaje de masas con un mínimo de recursos, de la primaria a la secundaria e incluyendo la formación a lo largo de toda la vida y la educación a distancia. De la voluntad política (políticas del sistema de ciencia y políticas del sistema de enseñanza) dependen las infraestructuras y el apoyo a programas de desarrollo (formación, cooperación internacional); y el financiamiento (que también importa) es reducido y previsible; es reducido sobre todo cuando se lo ecuaciona con las plusvalías (OCDE, 1998).

La globalización está ahí, a la vista, y en todos los niveles: la señal más visible será la economía global, la interdependencia de los mercados mundiales; las grandes organizaciones (ONU, Unesco, OCDE, Unicef, UE, Mercosur, etc.) constituyen grandes espacios político-económico-sociales indiscutibles, independientemente de su operacionalidad efectiva. Más próximos de las universidades, los programas de intercambio (Columbus, Sócrates, etc.) apuestan a la internacionalización de la formación a pesar de las restricciones de acceso a los niveles más elevados (Miranda, 1996; 1996).

¿Y el impacto en la psicología de la inteligencia humana?

Obviamente muy grande, con toda certeza. Al nivel de la investigación, la revisión crítica de los paradigmas y de 
los instrumentos de evaluación (Matarazzo, 1992), y el potencial de implicaciones incalculables abierto por los laboratorios virtuales. Al nivel de la intervención, la generalización de la formación a lo largo de la vida (Sternberg, 1997b) y una multitud de desafíos (muchos de ellos aún desconocidos) de la nueva realidad. A nivel de la enseñanza, alguna renovación conceptual, revolución de las prácticas, "alfabetización" informática, formación para la competitividad mundial.

¿Pero, será la sociedad del conocimiento y la globalización incondicionalmente un bien invalorable o un mal necesario? Ni una cosa ni la otra.

Estoy profundamente convencida del impacto positivo en la igualdad de oportunidades, en la aproximación entre los pueblos, en la universalidad de la democracia, en el derecho a la diferencia, en el desarrollo económico y en la promoción del bienestar.

Soy consciente de dos peligros: la uniformización, y el consecuente cultivo del conformismo. La sobrespecialización: durante años se ha luchado contra formar a Jack of all talents and master of none; llegó la hora de preparar Jack(s) of all talents although master of one ${ }^{9}$. La ingeniería (genética): al nivel de las consecuencias ¿podrá 1984 (la novela de G. Orwell escrita en

9 En lengua española, equivalente a "aprendiz de todo y maestro de nada" versus "aprendiz de todo a pesar de maestro de algo". el año 1949) venir a revelarse una profecía con error de fecha (en los años cuarenta el futuro parecía muy lejano...) ¿Por qué las comisiones de ética? Una única certeza: no nacieron por casualidad.

\section{¿Y el lugar de la esperanza?}

Ayer, como hoy y mañana, el humanismo integral y la educación para los valores. El humanismo como hilo conductor del desarrollo personal pleno en los mundos físico, social, cultural: del autoconocimiento y la autoestima a la preservación del ambiente, a la interiorización de los valores libertad-paztolerancia-solidaridad, pasando por la conciencia de la responsabilidad de todos y cada uno en la protección, transmisión y promoción del patrimonio cultural de la humanidad.

Albert Einstein (1879-1955), el físico genial y uno de los mayores humanistas de los tiempos modernos escribió un día:

Si el científico contemporáneo encontrara el tiempo y el valor para juzgar la situación y su responsabilidad (...) y actuara en función a esa evaluación, entonces las perspectivas de una solución razonable y satisfactoria, para la sumamente peligrosa situación internacional actual, surgirían profunda y radicalmente transformadas (Einstein, 1952/1979, p. 189).

No imagino discurso más pertinente $\mathrm{y}$ actual en las vísperas del siglo XXI. 


\section{REFERENCIAS}

Afonso, M.J. (1997, diciembre). "Successful Intelligence: um novo conceito, uma perspectiva abrangente de medida da inteligência". Ponencia presentada en la V Conferência Internacional de Avaliação Psicológica, Braga (Portugal). Mimeo.

Anastasi, A. (1997). Psychological testing (7a. ed.). Nueva York: Macmillan.

Barret, P.T. \& Eysenck, H.J. (1992). Brain evoked potential and intelligence. Intelligence, 16 (3-4), 361-381.

Binet, A. \& Henri, V. (1896). La psychologie individuelle. L'Année Psychologique, 2, 411-465.

Cattell, R.B. (1971). Abilities: their structure, growth and action. Boston: Houghton Mifflin.

Damásio, A.R. (1994). Descartes' error. Emotion, reason and human brain. Nueva York: Oxford University Press.

Damásio, A.R. (1999). The feeling of what happens. Body and emotion in the making of consciousness. Nueva York, Harcourt Brace \& Co.

Damásio, H. et al. (1994). The return of Phineas Gage: clues about the brain from the skull of a famous patient. Science, 264, 1102-05.

Einstein, A. (1979). Comment je vois le monde. París: Flammarion.

Feuerstein, R. (1979). Instrumental enrichment: an intervention program for cognitive modifiability. Baltimore: University Park.

Goleman, D. (1995). Emotional intelligence. Nueva York: Bantam Books.
Goleman, D. (1998). Working with emotional intelligence. Nueva York: Bantam Books.

Hebb, D.O. (1949). The organization of behavior. A neuropsychological theory. Nueva York: Wiley.

Huteau, M. \& Lautrey, J. (1999). Evaluer l'intelligence. Psychométrie cognitive. París: Presses Universitaires de France.

Lorenz, K. (1973) Die Rückseite des Spiegels. Munich: Piper Verlag.

Matarazzo, J.D. (1992). Psychological testing and assessment in the 21st century. American Psychologist, 47 (8), 10071018).

Matarazzo, J.D. (Ed.) (1972). Wechsler' measurement and appraisal of adult intelligence (5a. ed.). Baltimore: Williams \& Wilkins.

Marques, J.F. \& Miranda, M.J. (1996). Access to higher education in Portugal: selection procedures revisited, from studies at the University of Lisbon. Oxford Review of Education, 22 (3), 337-347.

Miranda, M.J. (1982). Exame do nivel intelectual das crianças portuguesas do Ensino Básico dos 6 anos 13 anos. Lisboa: Instituto Nacional de Investigação Científica.

Miranda, M.J. (1983). A amostragem de indivíduos. Revista Portuguesa de Pedagogia, XVII, 241-257.

Miranda, M.J. (1987). Perspectivas da investigação e avaliação da inteligência. Separata da Revista Portuguesa de Psicologia.

Miranda, M.J. (1994). Estudo do Inventário de Estilos de Pensamento com estudantes universitários: dados metrológicos. Psychologica, 12, 131-141. 
Miranda, M.J. (1996). Evaluation psychologique des élèves du Projet Plux: étude diférentielle longitudinale des aptitudes. En Estrela, A. (Coord.), Projet Plux, une expérience d'éducation interculturelle (93-106). Lisboa: Commission des Communautés Européennes/Faculdade de Psicologia e de Ciências da Educação.

Miranda, M.J. (1999). L'intelligence comme auto-gouvernement mental: du modèle à la mesure des styles cognitifs. En Huteau, M. \& Lautrey J. (Eds.), Psychologie et différences individuelles (pp. 197-200). Rennes: Presses Universitaires de Rennes.

Miranda, M.J. \& Marques, J.F. (1996). Sobre o acesso ao ensino superior em Portugal. Resultados e implicações de um estudo sectorial. Revista Ibero Americana de Diagnóstico y Evaluación Psicológica, 2 (2), 199-213.

Negroponte, N. (1996). Being digital. Nueva York: Vintage Books.

OCDE (1998). The global research village. París: Publications OCDE.

Oleron, P. (1989). L'intelligence de l'homme. París: Colin.

Piaget, J. \& Inhelder, B. (1970). La psychologie de l'enfant (4a. ed.). París: Presses Universitaires de France.

Piaget, J. (1956/1947). La psychologie de l'intelligence (4a. ed.). París: Colin.

Piaget, J. (1974). La prise de conscience. París: Presses Universitaires de France.

Reuchlin, M. (1995). Totalités, éléments, structures en psychologie. París: Presses Universitaires de France.

Sternberg, R.J. (1981). Nothing fails like success: the search for an intelligent paradigm for studying intelligence. Journal of Educational Psychology, 73, 142-155.

Sternberg, R.J. (1985). Beyond IQ: a triarchic theory of human intelligence. Cambridge: Cambridge University Press.

Sternberg, R.J. (1988). The triarchic mind: a new theory of human intelligence. Nueva York: Viking.

Sternberg, R.J. (1990). Metaphors of mind. Conceptions of the nature of intelligence. Cambridge: Cambridge University Press.

Sternberg, R.J. (1993). Triarchic abilities test. New Haven, CT: Yale University. Mimeo.

Sternberg, R.J. (1995). Defying the crowd. Nueva York: Free Press.

Sternberg, R.J. (1996). Successful intelligence. Nueva York: Simon \& Schuster.

Sternberg, R.J. (1997a). Thinking styles. Cambridge: Cambridge University Press.

Sternberg (Ed.) (1997b). Intelligence and lifelong learning special issue. American Psychologist, 52 (10).

Sternberg, R.J. \& Detterman, D.K. (Eds.) (1986). What is intelligence? Contemporary viewpoints on its nature and definition. Norwood: Ablex.

Thorndike, E.L. et al. (Eds.) (1921). Intelligence and its measurement: a symposium. Journal of Educational Psychology, 12, 123-147, 195-216.

Thorndike, E.L. et al. (1927). The measurement of intelligence. New York: Columbia University, Teachers College, Bureau of Publications. 
Vernon, P.E. (1960). Intelligence and attainment tests. Londres: University of London Press.

Vernon, P.E. (1961). The structure of human abilities. Londres: Methuen.

Vernon, P.E. (1979). Intelligence. Heredity and environment. San Francisco: Freeman.

Vygotsky, L.S. Mind in society: the development of higher psychological processes. Cambridge, MA: Harvard University Press.

Wechsler, D. (1958). The measurement and appraisal of adult intelligence (4a. ed.). Baltimore: Williams \& Wilkins.

Wechsler, D. (1975). Intelligence defined and undefined: a relativistic approach. American Psychologist, 30, 135-139. 